\title{
Early Warning Systems: Lost in Translation or Late by Definition? A FORIN Approach
}

\author{
Irasema Alcántara-Ayala ${ }^{1} \cdot$ Anthony Oliver-Smith ${ }^{2}$
}

Published online: 10 September 2019

(C) The Author(s) 2019

\begin{abstract}
Early warning systems (EWSs) are widely considered to be one of the most important mechanisms to prevent disasters around the globe. But as disasters continue to affect countries where EWSs have already been implemented, the striking disaster consequences have led us to reflect on the focus, architecture, and function of the warning systems. Since the 2004 Indian Ocean tsunami there has been a rapid rise in the promotion and use of EWSs to minimize disaster losses and damage. However, few researchers have addressed the question of their acceptability as an adaptive measure to the existing exposure conditions. EWSs are far more linked to emergency response and humanitarian crises and accepted technological interventions as solutions than they are to explicitly advance integrated analysis, disaster risk reduction, and policy making. A major flaw of EWSs is that the term "early" has been essentially used in reference to the speed of hazard onset, founded on a physicalist perspective that has encouraged a considerable dependence on technology. In this article we address the need for a clear understanding of the root causes and risk drivers of disaster risk creation, as advanced in the FORIN (forensic investigation of disasters) approach, as a prerequisite for the development of more articulated EWSs that could contribute to disaster risk reduction through policy making and practice, based on integrated and transdisciplinary management, in the interest of sustainable development, and human welfare and well-being.
\end{abstract}

Irasema Alcántara-Ayala

irasema@igg.unam.mx

1 Institute of Geography, National Autonomous University of Mexico (UNAM), 04510 Mexico City, Mexico

2 University of Florida, Gainesville, FL 32611, USA
Keywords Disaster risk drivers - Disaster root causes . Early warning systems · Forensic disaster investigations $\cdot$ FORIN

\section{Introduction: The Fallacy of Early Warning Systems}

The origin of early warning systems (EWSs) dates back to the 1980s, when famines in Sudan and Ethiopia generated the need to anticipate and avert future food crises (Kim and Guha-Sapir 2012). The consequences of the long socionatural processes of famine and drought raised the possibility of planning ahead. However, the evolution of the EWS concept and function has been diverted from its original conception in two ways. First, the definition of "early" is relative, according to natural hazard typology and speed of onset. From this we could assess whether or not the original sense of EWSs targeting actions well in advance has become irrelevant, but also whether current EWSs are already late by definition. We need to reflect on how "early" EWSs should be called on and made operational. Second, although there is no denying the significance of natural hazards in the definition of disaster risk, EWSs are mainly based on a physicalist perspective (Hewitt 1983a), where the emphasis is on the hazard, while social context is mostly neglected. Thus, the understanding of vulnerability and exposure within the "equation" of disaster risk remains unresolved.

Recognizing that EWSs are necessary can be regarded as a step into the sphere of social and institutional awareness and preparedness. It is clear, however, that enabling awareness and preparedness through EWSs requires not only the availability of scientific and practical information 
and resources to people, but also building capacity in terms of psychological and social capital to interpret and use information and resources according to local needs and expectations (Alcántara-Ayala et al. 2015), including the comprehensive understanding of disaster risk.

The current definition, architecture, and function of EWSs as an adaptive measure to the existing exposure conditions are not adequate. Disaster risk reduction (DRR) as the policy objective of disaster risk management (DRM) should encompass the social processes directed towards reducing existing disaster risk, managing residual risk, and especially avoiding the construction of new disaster risk in society (UNISDR 2017). The simple logic and common sense of EWSs should include the cognizance of disaster risk as a condition that is largely influenced by choices and decision making that lead to the misuse of land, derived from the socioeconomic practices promoting inequality that have led to the social construction of disaster risk and disasters. This would allow the systematic identification and analysis of their causal factors and drivers.

Based on proposals such as those developed by Basher (2006) and Wiltshire (2006), the most recent definition of EWSs provided by UNISDR (2017) emphasizes that to become "end-to-end" and "people-centered" EWSs need to include four interconnected key elements: "(1) disaster risk knowledge based on the systematic collection of data and disaster risk assessments; (2) detection, monitoring, analysis, and forecasting of the hazards and possible consequences; (3) dissemination and communication, by an official source, of authoritative, timely, accurate and actionable warnings and associated information on likelihood and impact; and (4) preparedness at all levels to respond to the warnings received" (UNISDR 2017, p. 14).

Nonetheless, there is also something of a dichotomy between current EWSs, which either mainly focus on the hazard component of disaster risk (WMO 2006; Rogers and Tsirkunov 2011), or follow a people-centered approach (Basher 2006; Thomalla and Larsen 2010), including the dissemination of information for the people to react to a given hazard, and those approaches that suggest that it is precisely the articulation of causality that should be the basic rationale of disaster risk reduction practice, a fundamental view provided by the Forensic Investigations of Disasters perspective (FORIN) (Oliver-Smith et al. 2016, 2017a, 2017b).

The following article is organized into six sections. Section 2 develops the FORIN perspective and Sect. 3 outlines the disaster risk interlinkages that are created by underlying causes and drivers of disaster risk and disasters, attached to the histories and culture of societies according to particular conditions occurring over time that have given rise to certain conditions of vulnerability and exposure. Section 4 discusses how the original meaning of the term "early" has been lost from its original conception, and how currently it is largely understood in terms of the speed of hazard onset from a physicalist angle. Section 5 addresses the analysis of the role of EWSs in decreasing disaster impacts as opposed to reducing disaster risk. By looking at the disaster triggered by Hurricane Katrina in 2005, we illustrate in Sect. 6 that the existing EWS at the time of the disaster was not sufficient for disaster risk reduction, and was only beneficial for groups at a lower level of vulnerability, as frequently seen in other disaster contexts. In the concluding section we reflect on the challenges of EWSs to incorporate the understanding of disaster risk and disasters as a process constructed by societies, in which vulnerabilities and exposure must be recognized and addressed as a baseline for solid DRM that could truly lead to DRR. The growing interest in the interface between integrated science and policy making (Cutter et al. 2015) also needs to stimulate contributions with respect to the possibility that EWSs could be implemented to reduce expenses and to avoid carrying out more structural forms of change while creating a false sense of security.

\section{Forensic Investigations of Disasters, FORIN}

The FORIN Project was established by the Integrated Research on Disaster Risk program of the International Science Council (IRDR-ISC) in 2010 (IRDR 2013). Its main objective is to increase and strengthen the knowledge that underlies evidence-based policy making for disaster risk management at all levels of governance and geographical scales. The insufficient understanding of the underlying or root causes of disasters, including their increased frequency and magnitude, is generally acknowledged in the worldwide disaster research, management, and policy communities. The FORIN Project is an international response (including both nongovernmental and intergovernmental organizations) to address this knowledge deficiency. The FORIN perspective formalizes the analytical space and agenda for root causality research, enabling a form of analysis that conceptualizes disasters as intrinsic to development and societal processes (Fig. 1) (Oliver-Smith et al. 2016, 2017a, 2017b).

The FORIN vision includes the idea of moving incrementally towards a forensic approach to the investigation of disasters triggered by geophysical, geomorphological, hydrometeorological, and technological events. Towards this end, the FORIN perspective proposes studying cases by using a common methodology in order to reach a broader and more probing analysis. These results, it is hypothesized, will go beyond the location- and timespecific findings that commonly emerge from single oneoff investigations and will help to identify underlying and 


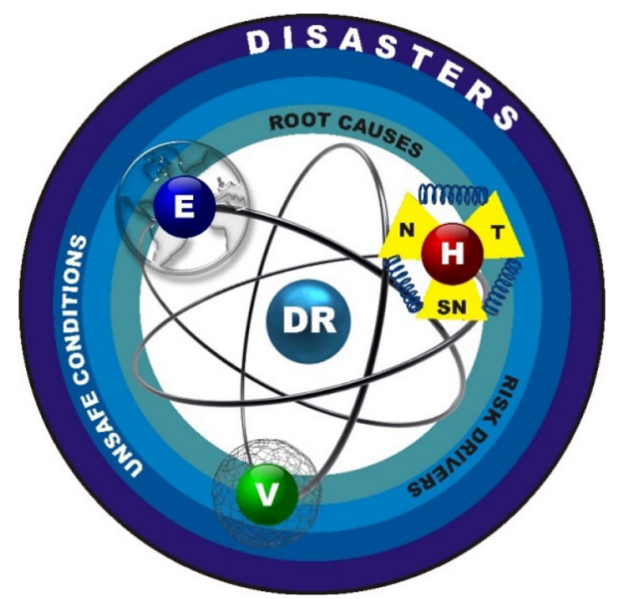

Fig. 1 Key relationships and processes in the social construction of risk. $E$ exposure, $V$ vulnerability, $H$ hazard, with the categories $N$ (natural), $T$ (technological), and $S N$ (socio-natural), $D R$ disaster risk (Oliver-Smith et al. 2016)

systemwide causes, allowing more effective disaster risk management, especially through the implementation of risk conscious sectoral and territorial development planning goals and mechanisms. The goal of the FORIN approach is the optimal design of the EWS component of the DRM/ DRR strategy.

FORIN research seeks to dispel the widespread but incorrect perception that disasters are independent events that happen in specific places to specific communities, where each event is perceived as unique and separate. FORIN postulates that disasters are linked both by systemic causes and by their widespread and expanding consequences in what can be termed an epidemiology of disasters (Burton 2010, 2015).

The complexity of disasters requires an interdisciplinary and holistic approach to research that is capable of capturing the multiple drivers, and interdependencies that combine to generate disasters. The organizational methodology provided by FORIN draws on joint, reciprocal framing of research questions, involving multiple stakeholders and multiple methodologies in the design, execution, and application of research leading to practice. Research on root causes will enable policymakers to trace causal trajectories in the construction of social vulnerability and the occupation of zones of high exposure to hazard, as well as environmental and social processes that are increasing risk that must be included when delineating EWSs.

Along these lines of thought, EWSs should be embraced in the dimension of the elementary shift of the understanding of disasters that involves moving beyond the erroneous and conceptually dangerous notion of "natural disasters" that pervades much political discourse. A new structure of EWSs is needed in order to advance development-based understanding of disaster risk construction as it concerns innovative organizational and institutional approaches based on scientific evidence and community participation. From the FORIN perspective it is clear that for identifying and analyzing disaster risk construction processes, a wide range of underlying causes and drivers linked to the social creation of disaster risk must be considered (Oliver-Smith et al. 2016, 2017a, 2017b).

Notwithstanding that both conceptually and practically EWS theorists and practitioners recognize the difficulty of changing the system and reducing vulnerability, in their present architecture, EWSs are more linked to emergency response and humanitarian crises than disaster risk reduction. Consequently, the present effort differs from the strategies devoted to using or further disseminating existing EWSs given that they are unquestioned technological interventions as adequate solutions rather than explicitly advancing integrated analysis, disaster risk research, and policy making. Most importantly, this article underscores the need for a clear understanding of root causes and risk drivers of disaster risk creation, as a prerequisite for the development of more articulated EWSs (Alcántara-Ayala and Oliver-Smith 2017) and better DRR and DRM. The latter should be delineated as a road map towards sustainable development, and human welfare and well-being (Lavell and Maskrey 2014).

\section{Root Causes, Histories, and Emerging Wisdom on Disaster Risk Interlinkages}

Since the beginning of civilization, in order to position themselves in the universe, human beings have developed philosophies, sciences, and myths about the relationship between people, nature, and the cosmos. In trying to understand Mother Nature and blaming the occurrence of catastrophes on fate or God's wrath for sins committed by humankind, disasters have been considered by many people around the world to be acts of gods and acts of nature (Hewitt and Burton 1971; O'Keefe et al. 1976; Burton et al. 1978; Hewitt 1983a, 1983b; Blaikie et al. 1994; Burton 2010, 2015; Oliver-Smith et al. 2016).

Much work on the significance of the cultural perception of disasters has been carried out in several regions of the world for emblematic cases such as the mudslides of Vargas, Venezuela in 1999 (Revet 2007), the 2001 Gujarat Earthquake in India (Simpson 2011), and the Vesuvius eruptions in Italy (Gugg 2018). An example of pioneering research includes the disaster triggered by the avalanche generated during the 1970 Ancash Earthquake in Peru, when approximately 70,000 people died (Oliver-Smith 1990). 
Bode $(1977,1989)$ and Oliver-Smith (1992) investigated the historical account of the cultural belief systems in the Peruvian Andes that constituted the constellation of cultural attitudes towards risk and decision making associated with disaster-driven displacement. Chester et al. (2008) documented the significance of religion in the perception of people in Italy towards volcanic eruptions and their effects, from a perspective of emergency response, for decision making framed by the window of the encounter between miracles and rationality.

Erroneously, disasters have been interpreted for a long time as the catastrophic inner self of the planet. The improvement of the understanding of disaster risk and its consequent shift of scientific paradigms has pointed to the unnaturalness of disasters as the line of reasoning that could shed light on disaster risk reduction and management (Alcántara-Ayala and Oliver-Smith 2017).

Over several decades, a growing body of literature has examined the different paradigms considered to explain disasters. In their seminal research, Burton, Kates, and Hewitt have led the discussion on the understanding of the interlinkages between environment and societies (Burton and Kates 1964; Burton et al. 1968, 1978; Hewitt and Burton 1971; Hewitt 1983a, 1983b) stating from the early stages that "Awareness of the risk of repeated disasters probably is higher in modern man, but the pattern of reinvasion of hazard areas is no less than in the past, and is very probably stronger" (Burton et al. 1968, p. 3).

As Maskrey (1993) argued, disasters are not natural. They are shaped by a series of processes of socioeconomic, historical, cultural, and institutional nature linked to development that have transformed the planet into a sphere of infinite dimensions of vulnerability and exposure (Hewitt and Burton 1971; Burton et al. 1978; Blaikie et al. 1994; Cannon 1994).

Denaturalizing disasters requires the conception of historical processes as disaster risk underlying causes and drivers, opposing the predominant vision of disasters as the equivalence of unavoidable "extreme" natural phenomena. Provided that risk must be understood as an integrated complex of conditions and variables that interact to place people at risk, the most important dimension and first stage to understanding disaster risk and disasters encompasses the recognition of root causes and risk drivers.

Influential contributions made by Blaikie et al. (1994), Cannon (1994), and Wisner et al. (2004) have placed emphasis on root causes as an interconnected series of widespread and general processes within a society, which can be distant in space, time, and essence as they are derived from culture, ideology, beliefs, social relations, and political economic systems prevailing over time. In many ways, the conception of the underlying causes of disasters mirrors the delineation of derived risk drivers or dynamic pressures. The latter are processes that embrace perceptions, choices, decision making, and actions that transform the spatial-temporal effects of root causes into unsafe conditions, and their infinite dimensionality of vulnerability, and therefore into disaster risks (Wisner et al. 2004).

The analysis of these phenomena is complex and must be addressed by engaging the complexity of the interactions of livelihoods, institutions, needs, priorities, perceptions, decision making, actions, resource allocation and production/consumption patterns, practices, and socioeconomic historical and contemporary developments within societies. Only through understanding these complex relationships can disaster risk interlinkages in terms of histories, emerging wisdom, and vulnerability be mapped.

It can be argued that interlinkages within political economic history at subnational, national, regional, and global scales-between population growth, urbanization, marginalization, inequity, poverty, exclusion, lack of adequate health and education conditions, migration, rural and urban land-use patterns, construction of infrastructure, environmental degradation and ecosystem service depletion, among other social asymmetries and exploitative types of social organization and misuse and mismanagement of natural resources and environment-have contributed to the skyrocketing expansion of vulnerable societies in areas of high exposure to the potential impact of hazards, and consequently to the increase of people at risk. These disaster risk drivers, as mechanisms and linkages that underpin the current conditions of societies in both developed and developing countries (Alcántara-Ayala 2002), have been generated from the implementation of systems of growth and development (Wisner et al. 2004; Oliver-Smith et al. 2016).

To address these linkages and the consequent spheres of vulnerability and exposure, the FORIN perspective creates an integrated framework for the drivers of disaster risk and the causality of disaster risk and disasters. The FORIN approach entails in-depth questioning and systemization of information concerning hazards, the exposed social and environmental domains, and the configuration of vulnerability within socioenvironmental frameworks. By considering relationships and the particular societal structures and organizations, FORIN aims at achieving a development process that is informed by DRM, bearing in mind its pivotal role for a transformational potential (Oliver-Smith et al. 2016).

In recent years there has been considerable interest in the FORIN perspective. It has become a critical issue in analyzing the infinite dimensions of vulnerability and exposure. By looking at 2009 Typhoon Morakot's devastation of Taiwan, Huang et al. (2013) questioned how people can better understand and learn from disasters, including the potential implications for emergency 
operations and disaster risk reduction if disasters are understood as socially constructed. Castillo (2013) addressed the integration of DRM with climate change adaptation in the Philippines, and Faustino-Eslava (2013) evaluated the necessity of the people to adjust their perception of their levels of exposure to potential hazards associated with the activity of an active fault system in the Philippines. More recent evidence highlights the influence of poor urban governance, politics, and institutional structures on the social construction of urban vulnerability to flooding by analyzing the root causes of flood vulnerability in the core area of metropolitan Ibadan, Nigeria (Salami et al. 2018).

\section{Lost in Translation}

The need to develop EWSs goes back to the African famine in Sudan and Ethiopia in 1984 and 1985 that resulted in the loss of life of around 1 million people (Kim and GuhaSapir 2012). Failure of the preceding early warning activities associated with the Sahel drought that led to famine, due to a lack of timely response even for a slow-onset phenomenon, produced considerable impacts with respect to human suffering, resources, and economic and social disruption (Nall and Josserand 1996).

Established in 1985 by the US Agency of International Development (USAID) and the US Department of State, the Famine Early Warning Systems (FEWS) was the first international effort created for relief ahead of time, identifying and working on the analysis of socio-natural processes, such as famines and droughts that could induce crisis and affect societies (FEWS NET 2019).

The principal aim of FEWS was focused on anticipating impending famines and generating advice for agency planners, policy making, and practice in terms of preventing or mitigating famines (Kim and Guha-Sapir 2012). To accomplish that purpose, field information and remote sensing imageries were used (Walsh 1986). However, just as the present day EWSs, FEWS faced obstacles associated with the limited availability of data and therefore with accuracy. Nonetheless, one of the major arguments employed to justify the creation of FEWS was precisely the duty to provide more than a general alarm. It was expected to convey specifics on location, numbers of people at risk, and other information regarding food security in the form of availability, access, and utilization (Walsh 1988).

Beyond the information derived from satellite observations, "the fact that man, not nature, is causing the problem poses special difficulties for relief organizers" (Walsh 1986 , p. 1145), a clear signal of the complex social challenges involved in EWSs. New attempts were directed toward the integration of physical and social data (Walsh 1986).

Initiatives in this direction were undertaken with the establishment of the International Decade for Natural Disaster Reduction (IDNDR) during the 1990s, particularly with respect to the need to understand disasters within a development context. A preamble event to the First World Conference on Natural Disaster Reduction (WCNDR) in Yokohama, Japan in 1994, the first Inter-American Conference on Reduction of Natural Disasters was held in Cartagena de Indias, Colombia. The main output of the event was the Cartagena Declaration on Reducing Vulnerability: A Goal of the Americas for Sustainable Development, which influenced the Yokohama Strategy for a Safer World: Guidelines for Natural Disaster Prevention, Preparedness and Mitigation and its Plan of Action (Lavell 2004; Alcántara-Ayala 2019).

One of the 10 outlined principles of the Yokohama Strategy pointed out that "Early warnings of impending disasters and their effective dissemination using telecommunications, including broadcast services, are key factors to successful disaster prevention and preparedness" (United Nations 1994, p. 8). This endeavor counted on incorporating "cost-effective technologies in reduction programs, including forecasting and warning systems" (United Nations 1994, p. 15) as a baseline for the Strategy for the year 2000 and beyond, and "establishing and/or strengthening early warning mechanisms for disaster reduction" (United Nations 1994, p. 16) as one of the cooperative activities to be encouraged at the regional and subregional levels.

The Hyogo Framework for Action 2005-2015 (HFA) also prioritized the identification, assessment, and monitoring of disaster risks and the enhancing of early warning (UNISDR 2005). Even before this strategy began, efforts concerning early warning had not been recognized as sufficient, as seen in the aftermath of the disaster associated with the 2004 Indian Ocean tsunami. Although up to this point the issue of early warning had been taken exclusively as a problem of developing countries, this myth was shattered by the devastating consequences of 2005 Hurricane Katrina. By considering the balance between the progress reported by countries and the occurrence of disasters during the execution period of the HFA, the specific gaps and challenges concerning risk identification, assessment, monitoring, and early warning prevailed. Perhaps the only difference was that during this time major disasters, including Hurricane Katrina and the Great East Japan Earthquake and Tsunami took place in the United States and Japan, respectively, two of the countries with the highest economic development, which were perceived as well prepared for confronting disasters. 
Parallel to the development of the IDNDR and HFA landmark agreements, FEWS evolved in 2000 into the FEWS NET, a network that currently provides information on and analysis of food insecurity based on an integrated approach that takes into account climate, agricultural production, prices, trade, nutrition, and the understanding of the character of local livelihoods, among others. The forecasts developed by the FEWS NET target potential scenarios and anticipate change 6-12 months in advance. A particular distinction of the FEWS NET is that it utilizes a livelihoods framework to characterize vulnerability and understand hazards according to geographical location (Boudreau 1998; Save the Children-UK 2000; Verdin et al. 2005); the latter is essential for identifying exposure.

The legacy of the devastating impact of the 2011 Great East Japan Earthquake and Tsunami (Fig. 2) is reflected in one of the seven global targets of the Sendai Framework for Disaster Risk Reduction 2015-2030 that was directed at substantially increasing "the availability of and access to multi-hazard early warning systems and disaster risk information and assessments to people by 2030" (UNISDR 2015 , p. 12) at global, regional, national, and local levels. In essence, this is aimed at investing in, developing, maintaining, and strengthening people-centered, multi- hazard approaches, as much as at multi-sectoral forecasting and early warning systems (UNISDR 2015).

The evolution and function of the concept of EWSs suggests that the term "early" has been mainly used in reference to the speed of onset according to hazard typology from a physicalist-centered perspective. The contemporary manifestation of this approach has fed a technologybased paradigm that has generated increasing vulnerability and exposure of communities.

Early warning systems, as originally conceptualized in FEWS, were characterized by a long-term perspective and analysis of the occurrence of events (that is, droughts, 6-12 months), which included historical investigation and the understanding of vulnerability as a condition, aiming at informing agency planners and policymakers. Current EWSs are focused on shorter-term occurrences of eventshurricanes (days), earthquakes (seconds); landslides (minutes, hours, days), volcanic eruptions (minutes, hours, days) (Fig. 3). They also lack a historical analytical perspective in the elaboration of EWSs, and hence do not consider root causes and disaster risk drivers. Vulnerability-if considered at all-is assumed from a static perspective, and the main goal is directed towards informing civil protection, but not towards DRR. Contemporary

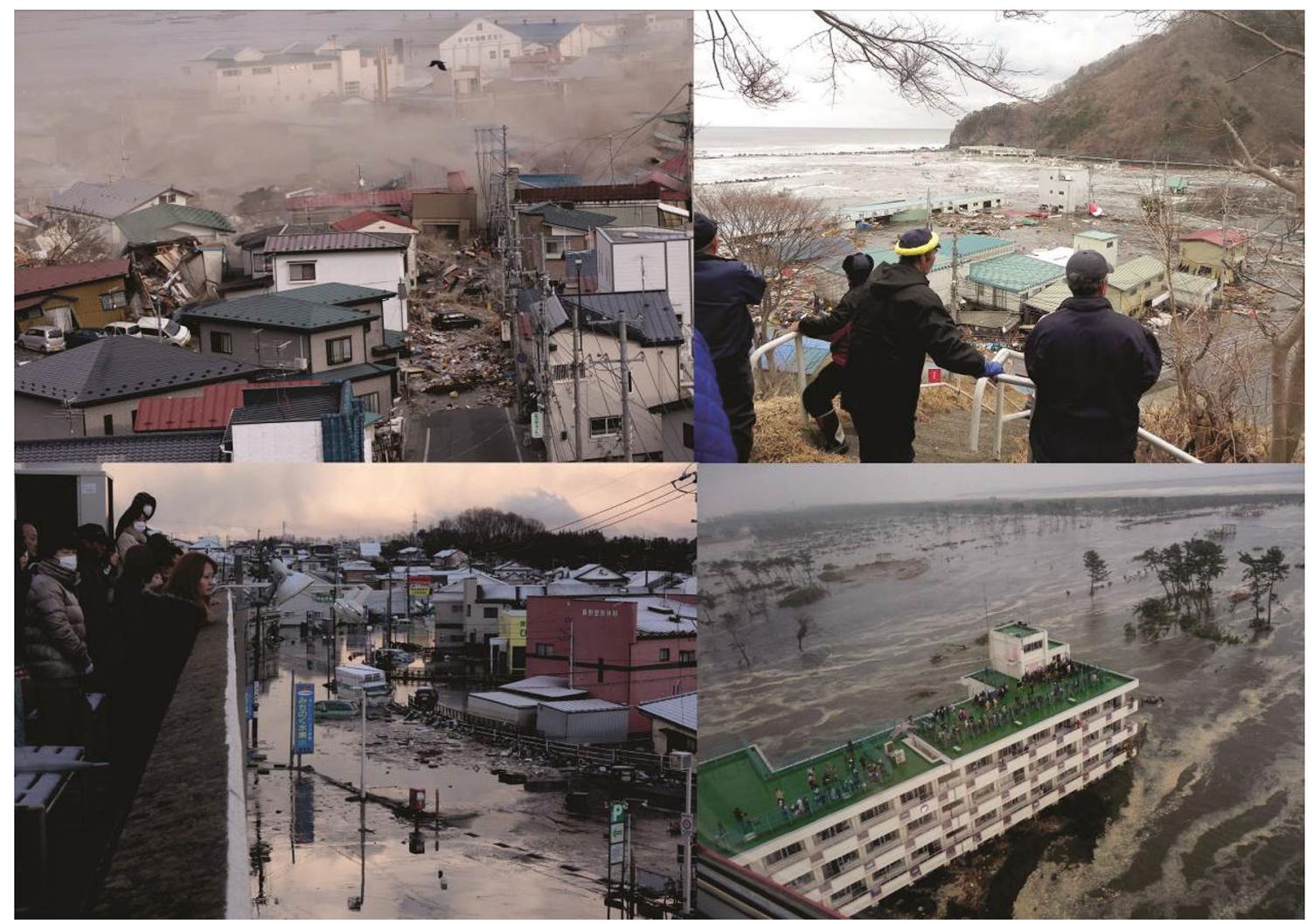

Fig. 2 Impacts of the 2011 Great East Japan Earthquake and Tsunami. Top left: Yamada Town, Iwate Prefecture. Top right: Fudai Village, Iwate Prefecture. Bottom left: Tagayo City, Miyagi
Prefecture. Bottom right: Sendai City, Miyagi Prefecture. Source Reproduced courtesy of the Tohoku Regional Bureau, Ministry of Land, Infrastructure, Transport and Tourism, Japan 


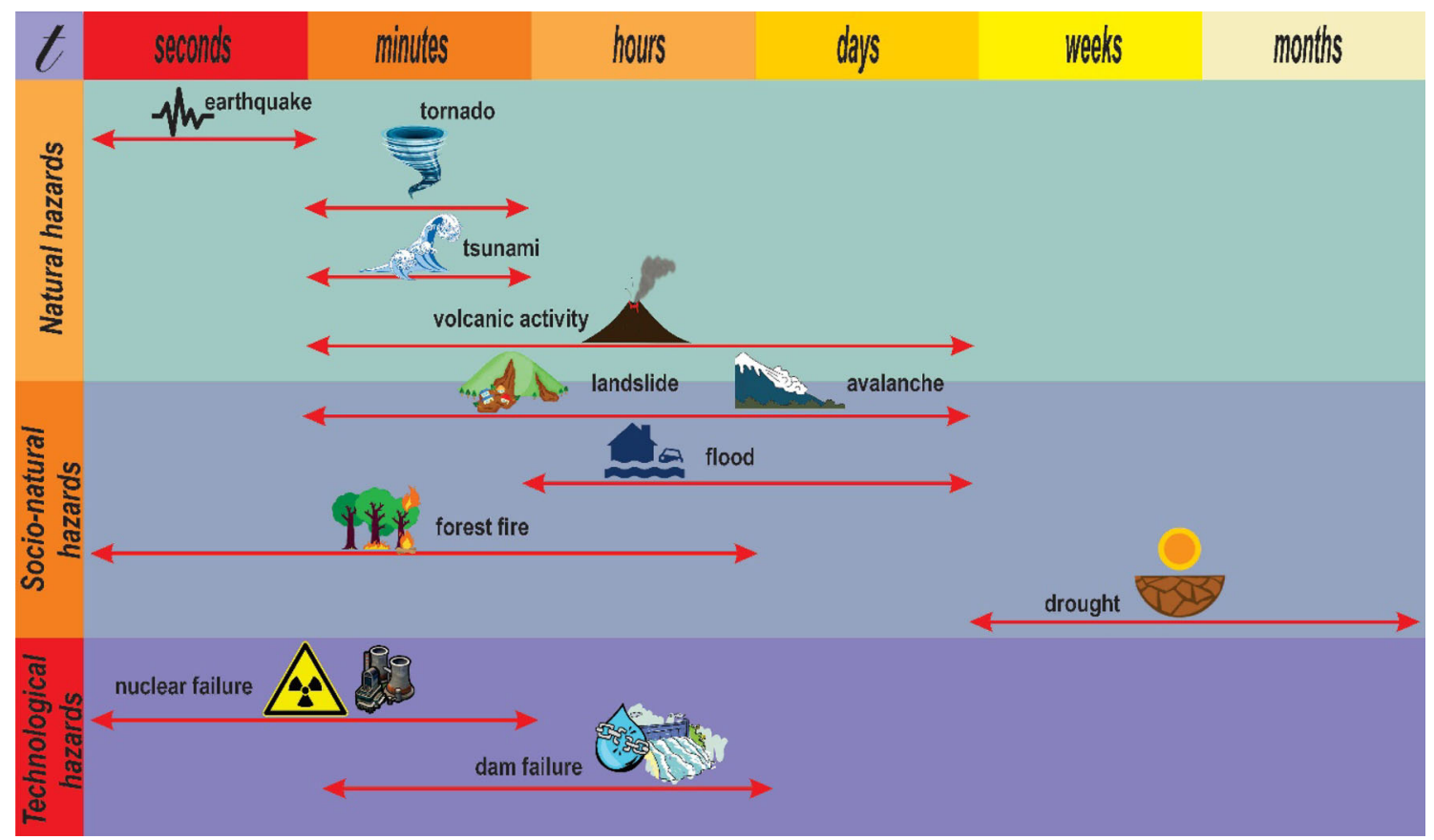

Fig. 3 Speed of hazard onset according to hazard typology from a physicalist-centered perspective. Early warning systems, as originally defined by famine early warning systems, were characterized by a long-term perspective and analysis of the occurrence of events

EWSs are focused primarily on reducing damages from hazard onset, rather than on reducing disaster risk. This suggests that scientists and technologists are more often than not the central stakeholders in early warning systems (Basher 2006).

While current EWSs provide some insights into certain aspects of response, they lack a critical approach to understanding how disaster risk is constructed. What has been lost in translation is the need to evolve into early warning articulated systems (EWASs) that could efficiently address DRR. In revisiting EWSs, a long-term perspective and analysis of events occurrence, from a FORIN angle, could contribute to translating root causes and disaster risk drivers as fundamental components for understanding disaster risk and for creating a new architecture for EWSs. If EWSs include vulnerability as a dynamic process in understanding that it often determines the magnitude of disasters, the rifts that have resulted from institutional vulnerabilities expressed in disasters in our societies could be taken as a source of lesson learning, and be aimed at informing both policymakers and civil protection agencies from a transdisciplinary perspective.

\section{Early Warning Systems: Disaster Impact Reduction Versus Disaster Risk Reduction}

Risk concerns uncertainty, which is a basic condition of human existence. When seeking DRR, the terms "risktaking" and "risk-aversion" or "risk-avoidance" involve different types of choices under uncertainty, and uncertainty can be reduced through learning and experience (Eiser 2004). Because disaster risk is rooted in the conditions of people living in an exposed area, risk avoidance does not necessarily involve changing conditions that generate risk but creating options or exits from the impacts of natural hazards. Current EWSs are on the wrong side of the scale because their progress has been assumed to be in the scope of disaster impact reduction as opposed to disaster risk reduction (Fig. 4).

Disaster risks are related and each of them contributes to the dynamic of the others, worsening and compounding disaster risk. Yet, the message of EWSs is focused on avoiding the consequences of detrimental exposure to nature and is often paired with claims about the difficulties of science in making accurate predictions of hazards, particularly under multi-hazard dynamics that encourage scientists to focus on the cause of "unusual" disasters. Perhaps the single greatest influence towards the integrated direction of EWSs should be the concept of DRR itself, as a process that changes the conditions that generate risk. The main failure of current EWSs is their focus on the hazard that triggers the disaster (Fig. 4). 


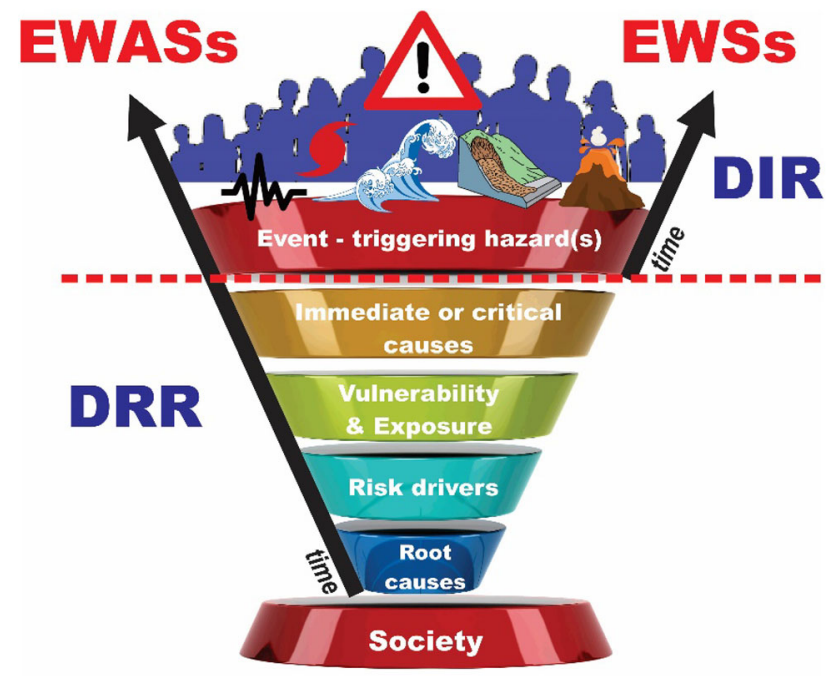

Fig. 4 Current early warning systems (EWSs) are merely focused on the hazard(s) as triggering mechanism(s) of disasters. Therefore, they contribute to disaster impact reduction (DIR), whereas early warning articulated systems (EWAS), from a FORIN perspective, call attention to the need for a clear understanding of the root causes and drivers of risk, as advanced in the FORIN approach in order to apply proper measures for disaster risk reduction (DRR)

The interpretation of peoples at risk very often involves concerns about the intensity of the threat, the expected magnitude, people's ability to cope, their fears and uncertainties, and their knowledge of the hazards that trigger the disaster. Although such factors have been recognized as influencing the effectiveness of EWSs (Twigg 2002), aspects associated with vulnerability and exposure commonly go unnoticed. Worrisome also is the fact that they are not the major concern for the people, though both determine the magnitude of disasters.

Individuals, as part of the environment within a socialecological system, have contributed to the construction of risk through choices made under institutional and social imposition, ignorance, and lack of information or perspective. The only difference of peoples at risk between countries and within countries is that they are located at different points-in socially constructed space and timealong the same disaster risk journey. Differences in terms of histories of failure derived from purely technological level-based EWSs are a matter of timing, as risk is largely institutionally conceived as a techno-scientific problem, and accordingly risk assessment is inclined to mask or ignore the underlying sociopolitical causes by foregrounding technical resolutions (Tucker and Nelson 2017).

The original conception of the EWSs considered both hazard dynamics and vulnerability, but as technology progressed, authorities recognized that forecasting with an acceptable degree of certainty the areas of impact of some hazards was possible, and EWSs have encouraged dependency on technology. In the aftermath of any disaster, the evident challenge is enhancing the capabilities of EWSs to avoid human and economic losses, whereas the desirable inclination would be to understand disasters as indicative of the "ills" of society and thus directing action towards addressing them.

Because territories, regardless of scale, are composed of different but not unique layers of vulnerability and exposure, large disasters in developed countries have rekindled the need to understand and manage disaster risk from a transdisciplinary perspective. The overall outcome of the day Hurricane Katrina struck New Orleans in 2005, for example, expressed some of the fundamental social processes considered as drivers of exposure and vulnerability (Oliver-Smith et al. 2016) that went beyond the southeastern region of the United States, raising questions about the nature of systems that perverted societies into becoming places of structured inequality, and therefore demanding diversity and social change.

The mounting impacts of disasters worldwide poses serious challenges to the transformation of EWSs into integrated systems. Basher (2006) underlined that integrated EWSs should include the connections among all the elements necessary for effective early warning and response, in particular the role of the human elements of the system and the management of risks instead of merely warning of hazards.

On a wider level, the definition, architecture, and function of EWSs should be rewritten by stimulating their transformation into early warning articulated systems (EWASs), composed of a coordinated structure with the capacity to contribute to the implementation of strategies of action to achieve DRR and DRM based on the understanding of disaster risk and disasters as a process constructed by societies.

As argued by Alcántara-Ayala and Oliver-Smith (2017), providing sound processes that sustain practices of close and permanent partnerships among all DRR stakeholders, and based on disaster risk-integrated science, EWASs should involve a responsible commitment to achieve and guarantee DRR and DRM in space and time. EWASs cannot be defined uniquely as a coordinating system of response, but most importantly they should provide the necessary elements for the comprehension of disaster risk as a social construct by considering the understanding of root causes of disasters, risk perception, and the different dimensions of vulnerability, resilience, and adaptation. The implementation of EWASs should be embedded in legal and ethical frameworks that rely on risk communication that aims at the construction of a culture of a risk conscious society, in order to avoid the potential incoherence of government policies and practices. 
The inadequacy of DRM and the policy manifested in the current architecture and function of EWSs, typified by a techno-centric and emergency response approach, is helping to undermine the road map towards DRR and sustainability. Within our contemporary societies, articulated EWSs, counter to our society's devotion and dependence on technological solutions, should be at the heart of the critical agenda for disaster risk reduction, while mere disaster impact reduction should be rejected.

\section{Hurricane Katrina: The Demise of a Myth}

The occurrence of Hurricane Katrina in 2005 in New Orleans and on the Gulf Coast triggered a disaster that involved the flooding of $80 \%$ of downtown New Orleans. The disaster resulted from the failure of dikes, storm surge, wind, and rain, and led to an estimated damage of USD 200 billion (Congleton 2006), more than half derived from the New Orleans flood (Boettke et al. 2007). Approximately 400,000 inhabitants were displaced, 1833 people lost their lives, and 500,000 people were affected overall (Geaghan 2011; CRED 2015).

The morphological attributes of the terrain where the city is located mostly below sea level, lacking natural drainage and adequate maintenance of floodwalls and levees for inundation control, were among the most significant elements that constrained the infrastructure capability as rainfall thresholds were also surpassed by Katrina (Alcántara-Ayala and Oliver-Smith 2017). This was strongly linked to the long-term alteration of the river dynamics and the deterioration of the Mississippi Deltaic Plain induced by human activities, including more than $15,000 \mathrm{~km}$ of canals dredged for oil and gas development, navigation, drainage, and logging, along with the construction of impoundments (Day et al. 2000, 2007). At rates of up to $100 \mathrm{~km}^{2} /$ year, about $4900 \mathrm{~km}^{2}$ of wetlands have been lost in coastal Louisiana since the beginning of the twentieth century (Day et al. 2007). This wetland degradation was one of the most significant risk drivers of the disaster.

The complexity of the disaster and the high magnitude of its impact responded to a series of conditions that emerged from a disproportionate dependence on technological protection, combined with an assault on the natural defenses of the environment of southern Louisiana (OliverSmith 2006). This relates to the "levee effect" that increases vulnerability to flooding as it builds a feeling of confidence over time that lessens preparedness and mitigation measures, and encourages the construction of infrastructure in hazardous zones, very often without proper maintenance (Pielke 1999; Kates et al. 2006; Burton and Cutter 2008).
These ideas are consistent with Burby (2006), in the emphasis on the use of safe development and local government complexities to explain the disaster associated with Hurricane Katrina. He argues that federal attempts to transform innately unsafe areas into secure areas by means of floodwalls and levees, linked to urbanization that lacked land-use planning, increased exposure conditions and people's vulnerability to hazards. At the same time, the meager attention and inadequate policy making regarding the potential disaster risk scenarios that result from the development of hazard-prone areas promoted by local governments also amplified vulnerability. Entrenched historical and political dynamics that lead to the construction of particular interrelationships of race and class as one of the main pivots of the creation of hazardscapes exhibit the complexity of the dimensions of vulnerability in societies in general (Bolin 2006) in both developed and developing countries.

As pointed out by Elliott and Pais (2006), when looking back into history, the uneven geographical development linked to the failure to develop strong commercial networks and industrial centers produced a peripheral region characterized by profound and complicated relations of race and class. Kates et al. (2006) traced back the configuration of exposure conditions within an historical account of 288 years-from the early stages of settlement on inequity in the location of African-American quarters in terms of potential flooding scenarios occurred.

Our perspective concurs also with that of Comfort et al. (2006) regarding the ineffective communication and coordination among agencies and levels of governmental jurisdiction, to identify adequate instruments and mechanisms for communication of fundamental information and coordination among key actors in the devastating conditions of Hurricane Katrina. However, this challenge has not been widely recognized. It is necessary to link EWSs with institutions, authorities, science, decision makers, and all other communities involved in DRR, as suggested by Alcántara-Ayala and Oliver-Smith (2017).

The EWS for Hurricane Katrina was not efficient for disaster impact reduction. It was only useful for decreasing the disaster risk mortality of groups with minor levels of vulnerability. African-American communities, characterized by an aged and ill population with a high level of poverty, were the most vulnerable, exposed, and affected by Katrina (Kates et al. 2006). One of the major drawbacks was the establishment of a top-down, command and control model of emergency management by the federal government after September 11, which lacked an intertwined vision and connection with agencies of emergency management at the local level. This raises the concern about the inclusion of the Federal Emergency Management Agency (FEMA) into the Department of Homeland Security 
(DHS), as seen in the increased institutional vulnerability of the system in the disaster triggered by Hurricane Katrina (Birkland 2009).

Katrina was a distinctive hurricane that "washed away any façade of racial and economic equality in America" (Reed 2007, p. 555). Beyond the extensive economic damage, the destruction of physical infrastructure, and the human and social disruption, including the psychological aftermath for the inhabitants and the displacement of over 400,000 residents (Geaghan 2011), Katrina exposed the vulnerabilities attached to the political system of the United States, including the poor and unnecessary duplicative coordination and communication between DHS, FEMA, and the different levels of governmental jurisdiction (Comfort 2006). The myth of the occurrence of major disasters as particularly characteristic of developing countries was also dispelled. Scenes of the disaster that were seen around the globe, "of desperate Black faces begging-for days-for rescue, for refuge, and for rations," were not scenes from the "third world" (Reed 2007, pp. 555-556). While disasters commonly affect the poorest and most marginalized people, their impact is not solely located in the most socioeconomically underprivileged countries (Blaikie et al. 1994; Cannon 1994); both developed and less developed countries are differentially vulnerable to hazards (Alcántara-Ayala 2002).

\section{Discussion and Conclusions}

Attempts to improve DRM at local, national, regional, and global scales have been addressed in several ways through international initiatives such as the Sendai framework for Disaster Risk Reduction 2015-2030, the current landmark agreement aiming at "the substantial reduction of disaster risk and losses in lives, livelihoods and health and in the economic, physical, social, cultural and environmental assets of persons, businesses, communities and countries" (UNISDR 2015, p. 12).

The Sendai Framework, based on Four Priorities of Action-(1) Understanding disaster risk; (2) Strengthening disaster risk governance to manage disaster risk; (3) Investing in disaster risk reduction for resilience; and (4) Enhancing disaster preparedness for effective response and to "Build Back Better" in recovery, rehabilitation and reconstruction (UNISDR 2015, p. 14)-seeks to attain its major goal by "preventing new and reducing existing disaster risk through the implementation of integrated and inclusive economic, structural, legal, social, health, cultural, educational, environmental, technological, political and institutional measures that prevent and reduce hazard exposure and vulnerability to disaster, increase preparedness for response and recovery, and thus strengthen resilience" (UNISDR 2015, p. 12).

Under such an agenda, understanding, recognizing, and addressing disaster roots or underlying causes went from being one of five basic priorities in the HFA (UNISDR 2005 ) to being one of 15 guiding principles in the Sendai Framework (UNISDR 2015). The pitfalls of the Sendai Framework, overlooking causality of disasters, pose a significant challenge, particularly for the importance of disaster risk knowledge, interpretation, and action in terms of organizational responses to disaster, because interpretations are shaped by experience, personal feelings and values, cultural beliefs, and interpersonal and societal dynamics (Eiser et al. 2012), but are not taken into consideration for the development of EWSs.

Disasters are products of an amalgam of complex disaster risk interlinkages that intersect the spheres of vulnerability, hazards, and exposure, which have unfolded and continue to unfold throughout history. As the understanding of the present is unavoidably linked to the past, it seems inescapable that addressing disaster risk and disasters is inseparable from the understanding of root causes and risk drivers that have led to the multifaceted dimensions of vulnerability and exposure, and hence of the social construction of risk.

In spite of the lack of quantitative assessments of the cost-benefit of EWSs in the medium and long terms, the way many people think about EWSs has been inspired by ideas about the importance of disaster loss avoidance, as well as by technologically driven views. However, disaster loss avoidance targeted by EWSs and visualized in terms of mortality and assets is far from synonymous with DRR and DRM.

In the absence of successful stories in both developed and developing countries, the pros and cons of EWSs have been progressively revealed by changes in EWS definition. Only a decade ago, EWSs were defined as "the set of capacities needed to generate and disseminate timely and meaningful warning information to enable individuals, communities and organizations threatened by a hazard to prepare and to act appropriately and in sufficient time to reduce the possibility of harm or loss" (UNISDR 2009, p. 12). Now, this concept has been changed to "an integrated system of hazard monitoring, forecasting and prediction, disaster risk assessment, communication and preparedness activities systems and processes that enables individuals, communities, governments, businesses and others to take timely action to reduce disaster risks in advance of hazardous events" (UNISDR 2017, p. 17).

Along these lines and anchored by a new conception of reducing disaster risk, EWSs would not be considered an integrated system if the notion of understanding the social construction of disaster risk is ignored. Even as the new 
DRR international agenda was consolidated in Sendai after the devastating disaster of the 2011 Great East Japan Earthquake and Tsunami, reflecting the need to "substantially increase the availability of and access to multi-hazard early warning systems [...] at global, regional, national and local levels" (UNISDR 2015, p. 12), by exclusively focusing on technology as the core of EWSs, disasters will continue to occur.

Rejecting the social construction and social understanding of disaster risk and disasters in favor of a mere technological approach will continue to bring disasters to our societies. The current architecture and function of EWSs, driven by a technological approach, reflects a single line of intent that is focused on the response to a potential disaster.

There is a genuine need for a rapprochement between the comprehensive understanding of disaster risk and the definition and establishment of EWSs. Underlying root causes and risks drivers should not be overlooked. We need to argue actively and persuasively against the definition and current function of EWSs as merely an adaptive measure to the existing exposure conditions, and in favor of bringing in an integrated disaster risk approach to show the policy relevance of the forensic investigations of disasters, the FORIN perspective directed towards the understanding of root causes and disaster risk drivers.

At this point in the recent history of disasters, when at the current rate every year disasters have an estimated impact of USD 520 billion and generate the displacement of millions of people, pushing 26 million inhabitants into poverty annually (UNISDR 2018), we wonder how lessons are easily lost, as increasing evidence of disaster impacts, and snowballing vulnerability and exposure conditions accrue.

As well as academic developments, changes outside academia have started to influence thinking. Societies are becoming more aware of the institutional vulnerabilities associated with the occurrence of disasters. A decade after the 2009 L'Aquila earthquake, in Italy there is evidence that disasters will become even more politicized and will enter legislative debates (Lauta 2018) in an era of human rights. A critical realist view of the drawbacks associated with the failures of both understanding disaster risk and disasters and current EWSs is indirectly documented through the cases seeking legal responsibility in the aftermath of disasters (Lauta 2014). More recent evidence, from an anthropological perspective, for example, highlights the need to go beyond the notion of strict legal frameworks and to investigate the symbolic, cultural, and social aspects of legal disputes associated with disasters, with the potential to reformulate cultural codes and transform social realities (Benadusi and Revet 2016).
Early warning systems should no longer ignore the root causes and risk drivers of disaster risk and disasters as they are intrinsically linked to the values and culture embedded in natural and social systems as unfolding processes over time (Oliver-Smith et al. 2016). Since the beginning of the international efforts on DRR, dating back to the establishment of the IDNDR, the strategies introduced into EWSs have failed to provide an understanding of the social construction of risk.

Recent disasters such as the 3 June 2018 volcanic eruption of Volcán de Fuego in Guatemala (Lavell and Brenes 2019), and the 28 September 2018 earthquake and tsunami in the city of Palu, Indonesia, demand an integrated disaster risk approach for policy making. The aftermath in the former included a death toll of 425 people, and an affected population of 714,414, whereas the latter accounted for 4340 fatalities, an affected population of 210,894 , and economic damage in the order of USD 1.45 billion (CRED 2019).

By looking at the root causes of the Volcán de Fuego disaster, Lavell and Brenes (2019) question why a possible volcanic event becomes a hazard and why there are specific existing conditions of vulnerability, all of them associated with territorial development, land use occupancy, livelihood schemes, and other socioeconomic and political processes. They suggest that in addition to a high level of dependency on external scientific sources for instrumentation and monitoring due to problems related to funding, the significance of the history of the affected Guatemalan towns and their inhabitants, and their antagonistic relationship with the government, and armed and security forces reflected the lack of formal risk management.

Owing to the consequences of the devastating disaster in the Indian Ocean triggered by the December 2004 tsunami, scientific research was directed towards the installation of a tsunami early warning system in Indonesia. Efforts made by the German-Indonesian Tsunami Early Warning System (GITEWS) project in 2005 (Rudloff et al. 2009) were the seeds for the establishment of the Indonesian Tsunami Early Warning Systems (InaTEWS) (UNESCO 2012). Inaugurated in 2008, and assessed in 2010 by an international commission, the system was handed over to the Indonesian government in 2011, and included training of the Indonesian staff in the use and maintenance of all components (Rakowsky et al. 2013).

Much concern has been expressed regarding the role of the warning center at the Indonesian Agency for Meteorology, Climatology, and Geophysics (BMKG), Jakarta, Indonesia in the aftermath of the disaster triggered by the tsunami associated with the earthquake of September 2018. The tsunami warning was lifted 34 min after issuing it, and according to Mr. Sutopo Purwo Nugroho, spokesman for the National Disaster Mitigation Agency (BNPB), the 
network of 22 buoys connected to seafloor sensors, needed to communicate advance tsunami warnings to the Indonesian Agency for Meteorology, Climatology, and Geophysics, had not worked since 2012 due to a lack of funding (ABC 2018).

In both the Guatemalan and Indonesian cases, the failure of EWSs is intertwined with the absence of financial and human resources, solid scientific development, capacity building, and the lack of political commitment and transdisciplinary science-based policy making - all well-defined indicators of some of the main dimensions of vulnerability that are not taken into consideration as key elements to comprehend the social construction of disaster risk. In spite of the efforts made by social scientists within the policymaking arena, DRR and DRM still tend to be dominated by technocratic decision making and practice, besides low public participation and accountability.

The effective engagement of FORIN, and its use at the level of practice, would lead to an integrated approach towards a new phase of policy-making structure and disaster risk culture. We hope that just as climate change is becoming a publicly recognized issue, the social construction of disaster risk could make headlines in newspapers, television, and social networks so that EWSs could be aligned with a new phase of relevance to policy making.

Disaster risk interlinkages in terms of vulnerability have been created in all societies within the sphere of multiple chronic endogenous and exogenous political and economic structures in association with religion, culture, perceptions, choices, authority regimes, development, and governance. In bringing social, economic, political, and cultural considerations that have unfolded through time into the ambit of disaster risk and challenging the predominance of the physicalist paradigm to explain disasters as social constructs, it will be possible to reveal the complexity of disaster risk reduction. This complexity is best expressed through identifying obstacles to reducing vulnerability and exposure in communities lacking sustainable development and solid and reliable disaster risk governance.

After several decades of false starts and neglect, the cumulative effect of disasters in the exponential growth of vulnerability and exposure in contemporary societies should compel the scientific community to engage in integrated disaster risk research, to begin to deal with EWSs and to avoid the radical dependency of EWSs on the dynamic of natural hazards. The lack of a central nucleus of disaster risk understanding in the existing architecture and function of EWSs serves more as a partial form of response than as a resolution to address disaster risk. Societies have thus continuously been affected by the need to be part of an integrated disaster risk perspective, very often at the expense of human casualties, and economic and environmental losses, in addition to conditions of vulnerability and exposure.

The momentum derived from recent disasters, where the implementation of EWSs was not sufficient to protect exposed societies, reflects the deep need of more cautious and integrated science to prevent "black swan" events. ${ }^{1}$ In practical terms, we strongly believe in the inclusion of current EWSs in the DRR international agendas, but the approach has often not been interdisciplinary or innovative. The current definition, architecture, and function of EWSs have tended towards technological responses that have little relation to the social context of their application. EWSs have not evaded a narrowing of their scope as they have freely withdrawn from framing understanding the significance of essential societal conditions of vulnerability and exposure, thus preventing them from participating in the search for an integrated reinvention. They are lost in translation, but also late by definition. From the dominance of technology and response, we must move through phases of disaster risk research to transdisciplinary integration and policy contribution.

Acknowledgements We would like to thank the Tohoku Regional Bureau, Ministry of Land, Infrastructure, Transport and Tourism, Japan, for the use of the photographs of the 2011 Great East Japan Earthquake and Tsunami disaster, and the Asian Disaster Reduction Center (ADRC) in Kobe. For Irasema Alcántara-Ayala to lead on this work, thanks are due to the PASPA-DGAPA, UNAM program (Support Program for the Improvement of Academic Personnel of the UNAM) for supporting this research. We are grateful to the reviewers and editors of the journal, who kindly provided suggestions to improve the manuscript.

Open Access This article is distributed under the terms of the Creative Commons Attribution 4.0 International License (http://crea tivecommons.org/licenses/by/4.0/), which permits unrestricted use, distribution, and reproduction in any medium, provided you give appropriate credit to the original author(s) and the source, provide a link to the Creative Commons license, and indicate if changes were made.

\section{References}

ABC. 2018. Indonesia tsunami early detection buoys haven't worked for six years due to "lack of funding". https://www.abc.net.au/ news/2018-10-01/indonesia-tsunami-early-detection-buoys-bro ken-for-six-years/10324200. Accessed 22 Apr 2019.

Alcántara-Ayala, I. 2002. Geomorphology, natural hazards, vulnerability and prevention of natural disasters in developing countries. Geomorphology 47(2-4): 107-124.

Alcántara-Ayala, I. 2019. Time in a bottle: Challenges to disaster studies in Latin America and the Caribbean. Disasters 43(S1): S18-S27.

Alcántara-Ayala, I., and A. Oliver-Smith. 2017. The necessity of early warning articulated systems (EWASs): Critical issues beyond

\footnotetext{
${ }^{1}$ A black swan event is an "unpredictable or unforeseen event, typically one with extreme consequences."
} 
response. In Identifying emerging issues in disaster risk reduction, migration, climate change and sustainable development, ed. K. Sudmeier-Rieux, M. Fernández, I.M. Penna, M. Jaboyedoff, and J.C. Gaillard, 101-124. Cham, Switzerland: Springer.

Alcántara-Ayala, I., O. Altan, D. Baker, S. Briceño, S. Cutter, H. Gupta, A. Holloway, A. Ismail-Zadeh, et al. 2015. Disaster risks research and assessment to promote risk reduction and management, ICSU-ISSC Ad Hoc Group on disaster risk assessment, ed. A. Ismail-Zadeh, and S. Cutter. Paris: ICSU. http://www.icsu. org/science-for-policy/disaster-risk/documents/DRRsynthesisPa per_2015.pdf. Accessed 28 Sept 2018.

Basher, R. 2006. Global early warning systems for natural hazards: Systematic and people-centred. Philosophical Transactions of the Royal Society A 364(1845): 2167-2182.

Benadusi, M., and S. Revet (eds.). 2016. On the witness stand: Environment crises, disasters and social justice. Archivio Antropologico Mediterraneo on Line-Anno XIX (2016), n. 18(2).

Birkland, T.A. 2009. Disasters, catastrophes, and policy failure in the homeland security era. Review of Policy Research 26(4): 423-438.

Blaikie, P., T. Cannon, I. Davis, and B. Wisner. 1994. At risk: Natural hazards, people's vulnerability, and disasters. London: Routledge.

Bode, B. 1977. Disaster, social structure and myth in the Peruvian Andes: The genesis of an explanation. Annals of the New York Academy of Sciences 293(1): 246-274.

Bode, B. 1989. No bells to toll: Destruction and creation in the Andes. New York: Scribners.

Boettke, P.J., E. Chamlee-Wright, P. Gordon, S. Ikeda, P. Leeson, and R. Sobel. 2007. The political, economic, and social aspects of Katrina. Southern Economic Journal 74(2): 363-376.

Bolin, B. 2006. Race, class, ethnicity, and disaster vulnerability. In Handbook of disaster research, ed. H. Rodríguez, E.L. Quarantelli, and R.R. Dynes, 113-129. New York: Springer.

Boudreau, T. 1998 The food economy approach: A framework for understanding rural livelihoods. Relief and rehabilitation network paper. London: Overseas Development Institute.

Burby, R.J. 2006. Hurricane Katrina and the paradoxes of government disaster policy: Bringing about wise governmental decisions for hazardous areas. Annals of the American Academy of Political and Social Science 604(1): 171-191.

Burton, C., and S.L. Cutter. 2008. Levee failures and social vulnerability in the Sacramento-San Joaquin Delta area, California. Natural Hazards Review 9(3): 136-149.

Burton, I. 2010. Forensic disaster investigations in depth: A new case study model. Environment: Science and Policy for Sustainable Development 52(5): 36-41.

Burton, I. 2015. The forensic investigation of root causes and the post-2015 framework for disaster risk reduction. International Journal of Disaster Risk Reduction 12: 1-2.

Burton, I., and R.W. Kates. 1964. The perception of natural hazard in resource management. Natural Resource Journal 3(3): 412-441.

Burton, I., R.W. Kates, and G. F. White. 1968. The human ecology of extreme geophysical events. FMHI Publications, Paper 78.

Burton, I., R.W. Kates, and G.F. White. 1978. The environment as hazard. New York: Oxford University Press.

Cannon, T. 1994. Vulnerability analysis and the explanation of natural disasters. In Disasters development and environment, ed. A. Varley, 13-30. Chichester: John Wiley.

Castillo, C. 2013. Harmonizing FORIN for climate change adaptation and disaster risk management to develop multi-sectoral narratives for Metro Manila. Project report. Quezon, Philippines: Manila Observatory.

Chester, D.K., A.M. Duncan, and C.J.L. Dibben. 2008. The importance of religion in shaping volcanic risk perception in Italy, with special reference to Vesuvius and Etna. Journal of Volcanology and Geothermal Research 172(3-4): 216-228.

Comfort, L.K. 2006. Cities at risk: Hurricane Katrina and the drowning of New Orleans. Urban Affairs Review 41(4): 501-516.

Congleton, R.D. 2006. The story of Katrina: New Orleans and the political economy of catastrophe. Public Choice 127(1-2): 5-30.

CRED (Centre for Research on the Epidemiology of Disasters). 2015. EM-DAT (Emergency Events Database). CRED, Université catholique de Louvain, Belgium. http://emdat.be/human_cost_ natdis. Accessed 22 Apr 2019.

CRED (Centre for Research on the Epidemiology of Disasters). 2019. EM-DAT (Emergency Events Database). CRED, Université catholique de Louvain, Belgium. https://www.emdat.be/data base. Accessed 22 Apr 2019.

Cutter, S.L., A. Ismail-Zadeh, I. Alcántara-Ayala, O. Altan, D.N. Baker, S. Briceño, H. Gupta, A. Holloway, et al. 2015. Global risks: Pool knowledge to stem losses from disasters. Nature 522(7556): 277-279.

Day, J.W., D.F. Boesch, E.J. Clairain, G.P. Kemp, S.B. Laska, W.J. Mitsch, K. Orth, H. Mashriqui, et al. 2007. Restoration of the Mississippi Delta: Lessons from Hurricanes Katrina and Rita. Science 315(5819):1679-1684.

Day, J.W., G. Shaffer, L. Britsch, D. Reed, S. Hawes, and D. Cahoon. 2000. Pattern and process of land loss in the Mississippi Delta: A spatial and temporal analysis of wetland habitat change. Estuaries 23(4): 425-438.

Eiser, J.R. 2004. Public perception of risk. Report prepared for Foresight Office of Science and Technology, London.

Eiser, J.R., A. Bostrom, I. Burton, D.M. Johnston, J. McClure, D. Paton, J. van der Pligt, and M.P. White. 2012. Risk interpretation and action: A conceptual framework for responses to natural hazards. International Journal of Disaster Risk Reduction 1: $5-16$.

Elliott, J.R., and J. Pais. 2006. Race, class, and Hurricane Katrina: Social differences in human responses to disaster. Social Science Research 35(2): 295-321.

Faustino-Eslava, D.V. 2013. Predictive forensics for averting possible disasters: A FORIN template for tackling issues related to the Valley Fault System and the Angat Dam in Luzon, Philippines. FORIN report. Laguna, Philippines: University of the Philippines, Los Banos.

FEWS NET (Famine Early Warning Systems Network). 2019. FEWS NET homepage. http://fews.net/. Accessed 28 Sept 2018.

Geaghan, K.A. 2011. Forced to move: An analysis of Hurricane Katrina movers 2009 American housing survey: New Orleans. SEHSD working paper number 2011-17, U.S. Census Bureau.

Gugg, G. 2018. Anthropology of the Vesuvius Emergency Plan: History, perspectives and limits of a dispositive for volcanic risk government. In Natural hazards and disaster risk reduction policies, ed. L. Antronico, and F. Marincioni, 105-123. Italy: Il Sileno Edizioni.

Hewitt, K. (ed.). 1983a. Interpretations of calamity from the viewpoint of human ecology. Boston: Allen \& Unwin.

Hewitt, K. 1983b. The idea of calamity in a technocratic age. In Interpretations of calamity from the viewpoint of human ecology, ed. K. Hewitt, 3-32. Boston: Allen \& Unwin.

Hewitt, K., and I. Burton. 1971. The hazardousness of a place: A regional ecology of damaging events. Research Publication 6, Department of Geography, University of Toronto, Toronto, Canada.

Huang, T., L. Hsiang-Chieh, Y. Hui-Hsuan, and L. Chung-Sheng. 2013. Towards a generic framework for synthesising the societal disturbance from Typhoon Morakot. Taipei: Science and Technology Center for Disaster Reduction. 
IRDR (Integrated Research on Disaster Risk). 2013. Integrated research on disaster risk strategic plan 2013-2017, Beijing, China. http://www.irdrinternational.org/wp-content/uploads/ 2013/04/IRDR-Strategic-Plan-2013-2017.pdf. Accessed 3 Sept 2019.

Kates, R.W., C.E. Colten, S. Laska, and S.P. Leatherman. 2006. Reconstruction of New Orleans after Hurricane Katrina: A research perspective. Proceedings of the National Academy of Sciences of the United States of America 103(40): 14653-14660.

Kim, J.J., and D. Guha-Sapir. 2012. Famines in Africa: Is early warning early enough? Global Health Action 5: Article 18481.

Lauta, K.C. 2014. Disaster law. Abingdon: Routledge.

Lauta, K.C. 2018. Disasters and responsibility. Normative issues for law following disasters. In: Disasters: Core concepts and ethical theories, ed. D. O’Mathúna, V. Dranseika, and B. Gordijn. Advancing Global Bioethics, vol. 11. Cham: Springer.

Lavell, A. 2004. LA RED: Background, training and contribution to the development of concepts, studies and practice in disaster risk and disasters in Latin America: 1980-2004 (La Red: Antecedentes, formación y contribución al desarrollo de los conceptos, estudios y la práctica en el tema de los riesgos y desastres en América Latina: 1980-2004). Panamá: La Red de Estudios Sociales en Prevención de Desastres en América Latina (in Spanish).

Lavell, A., and A. Brenes. 2019. Forensic analysis of the conditions of disaster risk in the 2018 Volcano of Fire (Volcán de Fuego) eruption. Opportunities for the strengthening of disaster risk management in Guatemala: A Study for the World Bank (preliminary version).

Lavell, A., and A. Maskrey. 2014. The future of disaster risk management. Environmental Hazards 13(4): 267-280.

Maskrey, A. (ed.). 1993. Disasters are not natural (Los desastres no son naturales). La Red de Estudios Sociales en Prevención de Desastres en América Latina, LA RED, Bogotá: Tercer Mundo Editores (in Spanish).

Nall, W., and H. Josserand. 1996. The USAID famine early warning system as an "information system". In Systèmes d'information et questions de développement, ed. J.P. Minvielle. Cahiers de Sciences Humaines 32(4): 903-911.

O'Keefe, P., K. Westgate, and B. Wisner. 1976. Taking the naturalness out of natural disaster. Nature 260(5552): 566-567.

Oliver-Smith, A. 1990. Post-disaster housing reconstruction and social inequality: A challenge to policy and practice. Disasters 14(1):7-19.

Oliver-Smith, A. 1992. The Martyred City: Death and rebirth in the Peruvian Andes, 2nd edn. Prospect Heights, IL: Waveland Press.

Oliver-Smith, A. 2006. Disasters and forced migration in the $21 \mathrm{st}$ century. http://understandingkatrina.ssrc.org/Oliver-Smith/. Accessed 28 Sept 2018.

Oliver-Smith, A., I. Alcántara-Ayala, I. Burton, and A. Lavell. 2016. Forensic Investigations of Disasters (FORIN): A conceptual framework and guide to research. IRDR FORIN publication No. 2. Beijing: Integrated Research on Disaster Risk, ICSU. http:// www.irdrinternational.org/wp-content/uploads/2016/01/FORIN21.pdf. Accessed 28 Sept 2018.

Oliver-Smith, A., I. Alcántara-Ayala, I. Burton, and A. Lavell. 2017a. Forensic Investigations of Disaster (FORIN): Towards the understanding of root causes of disasters. Policy brief. Beijing: Integrated Research on Disaster Risk, ICSU.

Oliver-Smith, A., I. Alcántara-Ayala, I. Burton, and A. Lavell. 2017b. The social construction of disaster risk: Seeking root causes. International Journal of Disaster Risk Reduction 22: 469-474.

Pielke, R.A. 1999. Nine fallacies of floods. Climatic Change 42(2): 413-438.

Rakowsky, N., A. Androsov, A. Fuchs, S. Harig, A. Immerz, S. Danilov, W. Hiller, and J. Schröter. 2013. Operational tsunami modelling with TsunAWI-recent developments and applications. Natural Hazards and Earth System Sciences 13(6): 1629-1642.

Reed, P.D. 2007. From the freedmen's bureau to FEMA: A postKatrina historical, journalistic, and literary analysis. Journal of Black Studies 37(4): 555-567.

Revet, S. 2007. Anthropology of a catastrophe: The mudslides of 1999 in Venezuela (Anthropologie d'une catastrophe: les coulées de boue de 1999 au Venezuela). Paris: Presses Sorbonne Nouvelle (in French).

Rogers, D., and V. Tsirkunov. 2011. Implementing hazard early warning systems. GFDRR WCIDS Report 11-03. https://www. preventionweb.net/files/24259_implementingearlywarningsys tems1108.pdf. Accessed 17 Apr 2019.

Rudloff, A., J. Lauterjung, U. Münch, and S. Tinti. 2009. Preface "The GITEWS project (German-Indonesian Tsunami Early Warning System)". Natural Hazards and Earth System Sciences 9(4): 1381-1382.

Salami, R.O., J. von Meding, and H. Giggins. 2018. The urban political ecology of flood vulnerability in the core area of Ibadan Metropolis, Nigeria. In Natural hazards and disaster risk reduction policies, ed. L. Antronico, and F. Marincioni, 36-50. Italy: Il Sileno Edizioni.

Save the Children-UK. 2000. The household economy approach: A resource manual for practitioners. London: Save the ChildrenUK.

Simpson, E. 2011. Blame narratives and religious reason in the aftermath of the 2001 Gujarat Earthquake, South Asia. Journal of South Asian Studies 34(3): 421-438.

Thomalla, F., and R.K. Larsen. 2010. Resilience in the context of tsunami early warning systems and community disaster preparedness in the Indian Ocean Region. Environmental Hazards 9(3): 249-265.

Tucker, B., and D.R. Nelson. 2017. What does economic anthropology have to contribute to studies of risk and resilience? Economic Anthropology 4(2): 161-172.

Twigg, J. 2002. The human factor in early warnings: Risk perception and appropriate communications. In Early warning systems for natural disaster reduction, ed. J. Zschau, and A.N. Küppers, 19-26. Berlin: Springer.

UNESCO (United Nations Educational, Scientific and Cultural Organization). 2012. Information guide: Tsunami early warning for broadcasting institutions in Indonesia. Pairs: UNESCO.

UNISDR (United Nations International Strategy for Disaster Reduction). 2005. Hyogo framework for action 2005-2015: Building the resilience of nations and communities to disasters. Geneva: UNISDR. https://www.unisdr.org/files/1037_hyogoframework foractionenglish.pdf Accessed 14 Apr 2019.

UNISDR (United Nations International Strategy for Disaster Reduction). 2009. Terminology on disaster risk reduction. http://www. unisdr.org/files/7817_UNISDRTerminologyEnglish.pdf. Accessed 14 Apr 2019.

UNISDR (United Nations International Strategy for Disaster Reduction). 2015. Sendai framework for disaster risk reduction 2015-2030. Geneva: UNISDR. http://www.preventionweb.net/ files/43291_sendaiframeworkfordrren.pdf. Accessed 17 Apr 2019.

UNISDR (United Nations International Strategy for Disaster Reduction). 2017. Report of the open-ended intergovernmental expert working group on indicators and terminology relating to disaster risk reduction. Geneva: UNISDR. https://www.preventionweb. net/files/50683_oiewgreportenglish.pdf. Accessed 14 Apr 2019.

UNISDR (United Nations International Strategy for Disaster Reduction). 2018. Sendai framework anniversary: Reporting underway. https://www.unisdr.org/archive/57495 Accessed 14 Apr 2019. 
United Nations. 1994. Yokohama strategy and plan of action for a safer world: Guidelines for natural disaster prevention, preparedness and mitigation. New York: United Nations. http:// www.unisdr.org/we/inform/publications/8241. Accessed $28 \mathrm{Sept}$ 2018.

Verdin, J., C. Funk, G. Senay, and R. Choularton. 2005. Climate science and famine early warning. Cautionary tales: Adaptation and the global poor. Philosophical Transactions of the Royal Society 360(1463): 2155-2168.

Walsh, J. 1986. Famine early warning closer to reality: Chronic lag in response to food emergencies in Africa spurs use of remote sensing to improve reaction time. Science 233(4769): $1145-1147$.
Walsh, J. 1988. Famine early warning systems wins its spurs. Science 239(4837): 249-250.

Wiltshire, A. 2006. Developing early warning systems: A checklist. In Proceedings of the 3rd International Conference on Early Warning (EWC III), 27-29 March 2006, Bonn, Germany.

WMO (World Meteorological Organization). 2006. Outcome report. Symposium on multi-hazard early warning systems for integrated disaster risk management, 23-24 May 2006, Geneva, Switzerland.

Wisner, B., P. Blaikie, T. Cannon, and I. Davis. 2004. At risk: Natural hazards, people's vulnerability and disasters, 2nd edn. New York: Routledge. 